\title{
Effect of Prosopis Farcta, Urtica Dioica and Disper root on Vegetative Growth, Nutrients Contents of cv. Shahreban Pomegranate Transplant
}

\author{
Azad A. Mayi ${ }^{1}$, Naji Isam Barwary ${ }^{2}$ (D) , Hasan Salim Nabi $^{3}$ \\ ${ }^{1,2,3}$ Department of Horticulture / College of Agricultural Engineering Science / University of Duhok. / \\ Kurdistan Region - Republic of Iraq \\ Corresponding Author: naji.isam@uod.ac
}

\begin{abstract}
Article history:
Received 31 March 2021

Accepted 08 May 2021
\end{abstract}

keyword : Pomegranate transplant, Prosopis

Farcta, Urtica Dioica , Disper root.

\begin{abstract}
This research was conducted on pomegranate transplants in a lath house during the growth season of 2020 of college of Agricultural engineering science, university of Duhok, to investigate the impact of spraying of Prosopis Farcta, Urtica Dioica and Disper root with 0,100 , and 200 mg.L-1 concentration, with 0,100 , and $200 \mathrm{mg} . \mathrm{L}-1$ concentration and with $(0,75$, and $150 \mathrm{mg} . \mathrm{L}-1)$ concentration respectively, on vegetative growth, nutrients contents of pomegranate transplants. The collected data indicate that the spraying of Prosopis Farcta extract, Urtica Dioica and Disper root especially at 200, 200, and $150 \mathrm{mg} . \mathrm{L}-1$ respectively resulting in a considerable increase in the majority of the examined parameters. At high concentrations, the interaction of three examined components resulted in the maximum values of branches number 14.57, transplant height $143.67 \mathrm{~cm}$, leaves number 157.33, Chlorophyll 48.97, Roots number 10, Root length $42.67 \mathrm{~cm}$, leaf nitrogen content $2.507 \%$, phosphorus $0.267 \%$ and potassium $1.433 \%$.
\end{abstract}

\section{Introduction}

The pomegranate (Punica granatum L.) is a member of the Punicaceae family of plants. It is one of the most widely consumed table fruits. This tree is native to Iran and has long been widely cultivated in the Mediterranean region (Sheikh and Manjula, 2009). Pomegranate trees can grow in a wide range of weather conditions, Pomegranate species don't need to be chilled very much. Frosts in the late spring do not hurt pomegranates, but frosts in the early fall can destroy the fruit in late varieties. Secondary metabolites can be found in fruit peels, leaves, tree stems, and root bark such as, dyes, tannins and alkaloids (Mirdehghan and Rahemi, 2007). It is critical to provide adequate nutrients to fruit trees throughout their first year of growth in order to aid in the development of their root systems and canopy structures and motivate a fast vegetative growth. Propagation, fertilization, irrigation, and other horticultural activities are only a few of the variables that aid growth of transplants of pomegranate. Currently, there is a global goal to limit the use of synthetic substances in crop production and to minimizing environmental pollution with human health impacts. Natural extracts for plants that are non-toxic, environmentally safe, and nutrient-dense, organic and costless are very important. The shrub Prosopis Farcta, is naturally grows in Iraq. The pods are considered a high-protein and high-carbohydrate food (Pasiecznik et al., 2004). Lajnef et al. (2015) reported that Prosopis Farcta seeds are easy to utilize and include protein and unsaturated fatty acids. And it contains flavonoids (DirekvandMoghadam et al., 2014). Poudineh et al. (2015) Showed that antioxidant activity is found in several parts of the plant (pods, seeds, and leaves). Nettle (Urtica Dioica L.) is a perennial wild plant that belongs to the Urticaceae family. The nettle stalks and leaves are considered to be a good source of vitamins $\mathrm{A}, \mathrm{B}$ and $\mathrm{C}$, minerals (calcium, iron, potassium, magnesium), and also pigments, especially carotenoids and chlorophyll (GuilGuerrero et al. 2003 ; Repajic et al. 2020). The roots nettle as well as contain a high source of sterols, protein lectin, polysaccharides, phenols and lignans (Sajfrtová et al.2005; Otles and Yalcin 2012; Orcic et al. 2014). Disper Root GS is a product that is specifically developed to promote root growth. 
Each Disper Root contain free amino acids (38.0 $\%)$, polysaccharides $(16.7 \%)$, vitamins (3.9\%), humic extract (14.0\%), phosphorus (3.7 \%), calcium $(1.2 \%)$, potassium $(3.5 \%)$ (www.edenmodemadriculture.com Spain).

The target of this study was response of Prosopis Farcta extract, Urtica Dioica and Disper root and their interactions on some vegetative growth parameters, nutrients contents of pomegranate transplant cv. Shahreban.

\section{Materials and Methods}

The experiment took place in the college of agricultural engineering science in the lath house; University of Duhok in the year 2020 on pomegranate transplant to investigate the effect of Prosopis Farcta extract, Urtica Dioica and Disper root and their interactions on the some vegetative growth parameters, nutrients contents of pomegranate transplant., The foliar spraying of the experiment elements took place twice. The first spray was in first week of April and second spray May 2020, first week. The effects of three levels of Prosopis Farcta extract were investigated in this study, 0, 100, and $200 \mathrm{mg} . \mathrm{L}-1$, with three levels of Urtica Dioica 0, 100, and $200 \mathrm{mg} . \mathrm{L}-1$, and Disper root 0,75 , and $150 \mathrm{mg} . \mathrm{L}-1$, Prosopis farcta fruit were collected from Sumel desert / Duhok, then dried, and powdered. 50 grams of crushed fruits was added in $1000 \mathrm{~mL}$ of ethanol $80 \%$ for twenty four hours at room temperature and then a filter paper was used to remove the impurities. To prevent the solvent from evaporating, the solution was stored in the oven for $1-2$ days at $40^{\circ} \mathrm{C}$. Urtica Dioica leaves were collected from Barwary bala / Duhok, and then it was dried for two days in the oven at $70^{\circ} \mathrm{C}$ then crushed. Fifty grams of powdered leaves was added in $1000 \mathrm{~mL}$ of ethanol $80 \%$ for 24 hours and then filter paper was used to filter the solution. The test was included of 27 interaction treatments and 3 replications; with (10) transplant individual and 270 transplant for each experimental unit and factorial Randomize Complete Block Design used (RCBD) with means calculated similar to Duncan's multiple range test at a portability rate of 5\% (Al-Rawi, and Khalaf-Alla, 2000) and the data was analyzed by utilize SAS (SAS Institute.2015) institute. The following are the parameters that were measured in this test; branches number (branch. transplant-1), transplant height $(\mathrm{cm})$, leaves number (leaves. shoot -1), Chlorophyll by using chlorophyll meter (SPAD), Roots number (root. transplant -1), Root length $(\mathrm{cm})$, The total nitrogen, phosphorus, and potassium percentages, digested dry plant leaf samples in acid were calculated as follows: The nitrogen estimated by using Microkjeldhal apparatus (A.O.A.C. 1975), Phosphorus was calculated using ammonium vanadium, and Spectrophotometer apparatus $(410 \mathrm{~nm})($ Olsen and Semmers 1982). The potassium concentration as determined by the Flame photometer equipment (Jenway PEP7, U.K.) provided by (page, et al, 1982 and A.O.A.C., 1995).

\section{Results and Discussion}

\section{1- Branches Number (Branch. Transplant-1)}

Table 1 obviously indicates that spraying of Prosopis Farcta extract, significant effect on the branches number (branch. transplant-1) compare to control. In the same table Urtica Dioica spray on pomegranate transplants significantly increase branches number (branch. transplant-1). In this table we also show spraying of Disper root significantly affected. We show in this table the interaction between Prosopis Farcta and Urtica Dioica affected significantly on the branches number (branch. transplant-1) the highest value 13.83. Regarding the interaction between Prosopis Farcta extract and Disper root in same table at the rate $200 \mathrm{ppm}, 150 \mathrm{ppm}$ respectively gave the highest value. Spraying of Urtica Dioica at 200 mg.L-1 with Disper root at 150 mg.L-1 significantly affected on the branches number (branch. transplant-1) of pomegranate transplants compare to control. The interactions of the three components are shown in Table 1, the highest value was registered from the interaction of $200 \mathrm{mg}$.L-1 Prosopis Farcta +200 mg.L-1 Urtica Dioica +150 mg.L-1 Disper root compared with the minimum value from $0 \mathrm{mg} . \mathrm{L}-1$ Prosopis Farcta +0 mg.L-1 Urtica Dioica + 0 mg.L-1 Disper root. 
Table 1. Effect of Prosopis Farcta extract, Urtica Dioica and Disper root and their interactions on branches number (branch. transplant-1) of $\mathrm{cv}$. Shahreban pomegranate transplant

\begin{tabular}{|c|c|c|c|c|c|c|}
\hline Prosopis Farcta & Disper root & $\begin{array}{c}\text { Urtica } \\
\text { Dioica } \\
0 \text { mg.L }{ }^{-1}\end{array}$ & $\begin{array}{c}\text { Urtica } \\
\text { Dioica } \\
100 \text { mg.L }^{-1}\end{array}$ & $\begin{array}{c}\text { Urtica } \\
\text { Dioica } \\
200 \text { mg.L } \mathbf{L}^{-1}\end{array}$ & $\begin{array}{c}\text { P.farcta } * \\
\text { Disper } \\
\text { root }\end{array}$ & $\begin{array}{c}\text { Prosopis } \\
\text { farcta }\end{array}$ \\
\hline \multirow{3}{*}{$0 \mathrm{mg} . \mathrm{L}^{-1}$} & $0 \mathrm{mg} . \mathrm{L}^{-1}$ & $3.93 \mathrm{o}$ & $5.40 \mathrm{n}$ & $5.50 \mathrm{n}$ & $4.94 \mathrm{~h}$ & \multirow{3}{*}{$6.27 \mathrm{c}$} \\
\hline & $75 \mathrm{mg} . \mathrm{L}^{-1}$ & $5.80 \mathrm{mn}$ & $6.601 \mathrm{~m}$ & $6.601 \mathrm{~m}$ & $6.33 \mathrm{~g}$ & \\
\hline & $150 \mathrm{mg} . \mathrm{L}^{-1}$ & $6.87 \mathrm{kl}$ & $7.80 \mathrm{jk}$ & $7.93 \mathrm{j}$ & $7.53 \mathrm{f}$ & \\
\hline \multirow{3}{*}{$100 \mathrm{mg} . \mathrm{L}^{-1}$} & $0 \mathrm{mg} . \mathrm{L}^{-1}$ & $9.20 \mathrm{i}$ & $9.90 \mathrm{hi}$ & $10.07 \mathrm{hi}$ & $9.72 \mathrm{e}$ & \multirow{3}{*}{$11.01 \mathrm{~b}$} \\
\hline & $75 \mathrm{mg} . \mathrm{L}^{-1}$ & $10.43 \mathrm{gh}$ & $11.63 \mathrm{ef}$ & $11.33 \mathrm{fg}$ & $11.13 \mathrm{~d}$ & \\
\hline & $150 \mathrm{mg} . \mathrm{L}^{-1}$ & $11.60 \mathrm{ef}$ & $11.90 \mathrm{~d}-\mathrm{f}$ & $13.03 \mathrm{bc}$ & $12.18 \mathrm{c}$ & \\
\hline \multirow{3}{*}{$200 \mathrm{mg} \cdot \mathrm{L}^{-1}$} & $0 \mathrm{mg} . \mathrm{L}^{-1}$ & $12.13 \mathrm{c}-\mathrm{f}$ & $12.63 \mathrm{ce}$ & $13.07 \mathrm{bc}$ & $12.61 \mathrm{c}$ & \multirow{3}{*}{$13.48 \mathrm{a}$} \\
\hline & $75 \mathrm{mg} . \mathrm{L}^{-1}$ & $12.93 \mathrm{~b}-\mathrm{d}$ & $13.90 \mathrm{ab}$ & $13.87 \mathrm{ab}$ & $13.57 \mathrm{~b}$ & \\
\hline & 150 mg. $\mathrm{L}^{-1}$ & $13.80 \mathrm{ab}$ & $14.40 \mathrm{a}$ & $14.57 \mathrm{a}$ & $14.26 \mathrm{a}$ & \\
\hline \multicolumn{2}{|c|}{ Urtica Dioica } & $9.63 \mathrm{~b}$ & $10.46 \mathrm{a}$ & $10.66 \mathrm{a}$ & \multirow{4}{*}{\multicolumn{2}{|c|}{ Disper root }} \\
\hline \multirow{3}{*}{$\begin{array}{l}\text { Prosopis Farcta } \\
* \text { Urtica Dioica }\end{array}$} & $0 \mathrm{mg} \cdot \mathrm{L}^{-1}$ & $5.53 \mathrm{f}$ & $6.60 \mathrm{e}$ & $6.68 \mathrm{e}$ & & \\
\hline & $100 \mathrm{mg} \cdot \mathrm{L}^{-1}$ & $10.41 \mathrm{~d}$ & $11.14 \mathrm{c}$ & $11.48 \mathrm{c}$ & & \\
\hline & $200 \mathrm{mg} \cdot \mathrm{L}^{-1}$ & $12.96 \mathrm{~b}$ & $13.64 \mathrm{a}$ & $13.83 \mathrm{a}$ & & \\
\hline \multirow{3}{*}{$\begin{array}{l}\text { Urtica Dioica } \\
\text { *Disper root }\end{array}$} & $0 \mathrm{mg} . \mathrm{L}^{-1}$ & $8.42 \mathrm{~d}$ & $9.31 \mathrm{c}$ & $9.54 \mathrm{c}$ & $0 \mathrm{mg} . \mathrm{L}^{-1}$ & $9.09 \mathrm{c}$ \\
\hline & $75 \mathrm{mg} . \mathrm{L}^{-1}$ & $9.72 \mathrm{c}$ & $10.71 \mathrm{~b}$ & $10.60 \mathrm{~b}$ & $75 \mathrm{mg} . \mathrm{L}^{-1}$ & $10.34 \mathrm{~b}$ \\
\hline & $150 \mathrm{mg} . \mathrm{L}^{-1}$ & $10.76 \mathrm{~b}$ & $11.37 \mathrm{a}$ & $11.84 \mathrm{a}$ & $150 \mathrm{mg} . \mathrm{L}^{-1}$ & $11.32 \mathrm{a}$ \\
\hline
\end{tabular}

Means within a row, column and their interactions, followed with the same letters are not significantly different from each other according to Duncan's multiple ranges test at significant level of $5 \%$.

\section{2- $\quad$ Transplant Height (cm)}

In this table recorded that spraying of Prosopis Farcta had significant effect on the transplant height $141.60 \mathrm{~cm}$. Table 2 clearly shows that spraying of Urtica Dioica with 200 mg.L-1 significantly affected transplant height, the highest value 132.26. In the table 2 recorded that spraying of Disper root with 150 mg.L-1 significantly affected on transplant the highest value $134.67 \mathrm{~cm}$. The effects of combination of Prosopis Farcta and Urtica Dioica were shown in table 2. Pomegranate transplants treated with $200 \mathrm{mg} . \mathrm{L}-1$ of both provided the most substantial value compare to control have the lowest value. The interaction of Prosopis Farcta and Disper root affected significantly on transplant height with $200 \mathrm{mg}$.L-1, $150 \mathrm{mg} . \mathrm{L}-1$ compare to interaction of both with 0 mg.L-1, 0 mg.L-1 respectively that gave lowest value. As shown in the table 2 the combination among Urtica Dioica and Disper root also affected significantly on transplant height of Pomegranate cv. Shahreban, since the most value was took from treatment combination $200 \mathrm{mg} . \mathrm{L}-1$ Urtica Dioica with $150 \mathrm{mg} . \mathrm{L}-1$ Disper root. Table 2 depicts the interactions of three factors differed significantly; the maximum value $144.00 \mathrm{~cm}$ was recorded from the combinations of $200 \mathrm{mg}$.L-1 Prosopis Farcta +100 mg.L-1 Urtica Dioica + 150 mg.L-1 Disper root evaluate with the minimum value $101.67 \mathrm{~cm}$ from the mixture of $0 \mathrm{mg} . \mathrm{L}-1$ Prosopis Farcta +0 mg.L-1 Urtica Dioica + 0 mg.L-1 Disper root.

Table 2. Effect of Prosopis Farcta extract, Urtica Dioica and Disper root and their interactions on transplant height $(\mathrm{cm}) \mathrm{cv}$. Shahreban pomegranate transplant

\begin{tabular}{|c|c|c|c|c|c|c|}
\hline $\begin{array}{c}\text { Prosopis } \\
\text { Farcta }\end{array}$ & $\begin{array}{c}\text { Disper } \\
\text { root }\end{array}$ & $\begin{array}{l}\text { Urtica Dioica } \\
0 \text { mg.L }\end{array}$ & $\begin{array}{c}\text { Urtica } \\
\text { Dioica } \\
100 \text { mg.L }^{-1}\end{array}$ & $\begin{array}{c}\text { Urtica } \\
\text { Dioica } \\
200 \text { mg.L } \text {-1 }^{-1}\end{array}$ & $\begin{array}{c}\text { P.farcta } * \\
\text { Disper root }\end{array}$ & $\begin{array}{c}\text { Prosopi } \\
s \\
\text { farcta }\end{array}$ \\
\hline \multirow{3}{*}{$0 \mathrm{mg} \cdot \mathrm{L}^{-1}$} & $0 \mathrm{mg} \cdot \mathrm{L}^{-1}$ & $101.67 \mathrm{k}$ & $107.80 \mathrm{j}$ & $114.03 \mathrm{i}$ & $107.83 \mathrm{~g}$ & \multirow{3}{*}{$115.75 \mathrm{c}$} \\
\hline & $75 \mathrm{mg} . \mathrm{L}^{-1}$ & $116.57 \mathrm{hi}$ & $116.27 \mathrm{hi}$ & $118.70 \mathrm{gh}$ & $117.18 \mathrm{f}$ & \\
\hline & $150 \mathrm{mg.L}^{-1}$ & $119.00 \mathrm{gh}$ & $121.40 \mathrm{~g}$ & $126.33 \mathrm{f}$ & $122.24 \mathrm{e}$ & \\
\hline \multirow{3}{*}{$100 \mathrm{mg} \cdot \mathrm{L}^{-1}$} & $0 \mathrm{mg} . \mathrm{L}^{-1}$ & $131.60 \mathrm{e}$ & $126.57 \mathrm{f}$ & $131.20 \mathrm{e}$ & $129.79 \mathrm{~d}$ & \multirow{3}{*}{$133.45 \mathrm{~b}$} \\
\hline & $75 \mathrm{mg} . \mathrm{L}^{-1}$ & $130.43 \mathrm{e}$ & $132.03 \mathrm{e}$ & $134.00 \mathrm{de}$ & $132.16 \mathrm{c}$ & \\
\hline & $150 \mathrm{mg} . \mathrm{L}^{-1}$ & $136.67 \mathrm{~cd}$ & $138.43 b c$ & $140.10 \mathrm{a}-\mathrm{c}$ & $138.40 \mathrm{~b}$ & \\
\hline \multirow{3}{*}{$200 \mathrm{mg} \cdot \mathrm{L}^{-1}$} & $0 \mathrm{mg} . \mathrm{L}^{-1}$ & $138.37 \mathrm{bc}$ & $140.30 \mathrm{a}-\mathrm{c}$ & $139.97 \mathrm{a}-\mathrm{c}$ & $139.54 \mathrm{~b}$ & \multirow{3}{*}{$141.60 \mathrm{a}$} \\
\hline & $75 \mathrm{mg} . \mathrm{L}^{-1}$ & $141.63 \mathrm{ab}$ & $141.67 \mathrm{ab}$ & $142.33 \mathrm{ab}$ & $141.88 \mathrm{a}$ & \\
\hline & $150 \mathrm{mg} . \mathrm{L}^{-1}$ & $142.43 \mathrm{ab}$ & $144.00 \mathrm{a}$ & $143.67 \mathrm{a}$ & $143.37 \mathrm{a}$ & \\
\hline
\end{tabular}




\begin{tabular}{|c|c|c|c|c|c|c|}
\hline \multicolumn{2}{|c|}{ Urtica Dioica } & \multirow{2}{*}{$\begin{array}{l}128.71 \mathrm{~b} \\
112.41 \mathrm{f} \\
\end{array}$} & \multirow{2}{*}{$\frac{129.83 \mathrm{~b}}{115.16 \mathrm{e}}$} & \multirow{2}{*}{$\frac{132.26 \mathrm{a}}{119.69 \mathrm{~d}}$} & \multirow{4}{*}{\multicolumn{2}{|c|}{ Disper root }} \\
\hline \multirow{3}{*}{$\begin{array}{c}\text { Prosopis } \\
\text { Farcta } \\
* \text { Urtica } \\
\text { Dioica }\end{array}$} & $0 \mathrm{mg} . \mathrm{L}^{-1}$ & & & & & \\
\hline & $100 \mathrm{mg} . \mathrm{L}^{-1}$ & $132.90 \mathrm{c}$ & $132.34 \mathrm{c}$ & $135.10 \mathrm{~b}$ & & \\
\hline & $200 \mathrm{mg} . \mathrm{L}^{-1}$ & $140.81 \mathrm{a}$ & $141.99 \mathrm{a}$ & $141.99 \mathrm{a}$ & & \\
\hline Urtica & $0 \mathrm{mg} . \mathrm{L}^{-1}$ & $123.88 \mathrm{f}$ & $124.89 \mathrm{f}$ & $128.40 \mathrm{e}$ & $0 \mathrm{mg} . \mathrm{L}^{-1}$ & $125.72 \mathrm{c}$ \\
\hline Dioica & $75 \mathrm{mg} . \mathrm{L}^{-1}$ & $129.54 \mathrm{de}$ & $129.99 \mathrm{de}$ & $131.68 \mathrm{~cd}$ & $75 \mathrm{mg} . \mathrm{L}^{-1}$ & $130.40 \mathrm{~b}$ \\
\hline *Disper root & $150 \mathrm{mg} . \mathrm{L}^{-1}$ & $132.70 \mathrm{bc}$ & $134.61 \mathrm{~b}$ & $136.70 \mathrm{a}$ & $150 \mathrm{mg} \cdot \mathrm{L}^{-1}$ & $134.67 \mathrm{a}$ \\
\hline
\end{tabular}

Means within a row, column and their interactions, followed with the same letters are not significantly different from each other according to Duncan's multiple ranges test at significant level of 5\%.

\section{3- $\quad$ Leaves Number (Leaves . Shoot - 1)}

Spraying of Prosopis Farcta was shown in Table 3 to be effective spray with 200 mg.L-1 significantly effect on leaves number (leaves. shoot -1) compared to control. Spraying process for both Urtica Dioica and Disper root with $200 \mathrm{mg} . \mathrm{L}-1$ and 150 mg.L-1 respectively gave highest leaves number (leaves. shoot -1) on pomegranate transplant compared to no spraying. Same table shows that the Prosopis Farcta was combined with Urtica Dioica. The interaction of both had significant effect on leaves number (leaves. shoot 1); compare to no spraying that gave lowest value. For the intention table 3 indicated that interactions of Prosopis Farcta + Disper root significantly differed by spraying $200 \mathrm{mg}$.L-1 and $150 \mathrm{mg}$.L-1 respectively on leaves number (leaves. shoot -1) of pomegranate shehreban cultivar. Concerning the interactions of all studied factors, results in this table demonstrate that the maximum value of number of leaves (shoot -1), 149.33 was recorded from the interactions of $200 \mathrm{mg}$.L-1 Urtica Dioica and $150 \mathrm{mg} . \mathrm{L}-1$ Disper root compared to the interactions 0 mg.L-1 Urtica Dioica and 0 mg.L-1 Disper root that recorded the lowest value 138.56. In terms of three-factor interactions, results indicated that the maximum value of leaves number (leaves. shoot -1), 157.33 was registered from the interactions of $200 \mathrm{mg} . \mathrm{L}-1$ Prosopis Farcta +200 mg.L-1 Urtica Dioica + 150 mg.L-1 Disper root compared to the control 121.33.

Table 3. Effect of Prosopis Farcta extract, Urtica Dioica and Disper root and their interactions on leaves number (leaves. shoot -1) of cv. Shahreban pomegranate transplant

\begin{tabular}{|c|c|c|c|c|c|c|}
\hline $\begin{array}{l}\text { Prosopis } \\
\text { Farcta }\end{array}$ & Disper root & $\begin{array}{c}\text { Urtica } \\
\text { Dioica } \\
\text { 0 mg.L }{ }^{-1}\end{array}$ & $\begin{array}{c}\text { Urtica } \\
\text { Dioica } \\
100 \mathrm{mg} . \mathrm{L}^{-1}\end{array}$ & $\begin{array}{c}\text { Urtica } \\
\text { Dioica } \\
200 \mathrm{mg} . \mathrm{L}^{-1}\end{array}$ & $\begin{array}{l}\text { P.farcta } * \\
\text { Disper root }\end{array}$ & $\begin{array}{c}\text { Prosops } \\
\text { farcta }\end{array}$ \\
\hline \multirow{3}{*}{$0 \mathrm{mg} \cdot \mathrm{L}^{-1}$} & $0 \mathrm{mg} . \mathrm{L}^{-1}$ & 121.331 & $125.67 \mathrm{kl}$ & $127.67 \mathrm{k}$ & $124.89 \mathrm{~g}$ & \multirow{3}{*}{$133.15 \mathrm{c}$} \\
\hline & $75 \mathrm{mg} . \mathrm{L}^{-1}$ & $129.67 \mathrm{k}$ & $136.00 \mathrm{j}$ & $138.00 \mathrm{ij}$ & $134.56 \mathrm{f}$ & \\
\hline & 150 mg. $\mathrm{L}^{-1}$ & $139.67 \mathrm{~h}-\mathrm{j}$ & $138.67 \mathrm{ij}$ & 141.67 & $140.00 \mathrm{e}$ & \\
\hline \multirow{3}{*}{$100 \mathrm{mg} \cdot \mathrm{L}^{-1}$} & $0 \mathrm{mg} \cdot \mathrm{L}^{-1}$ & $144.33 \mathrm{f}-\mathrm{h}$ & $145.67 \mathrm{e}-\mathrm{g}$ & $148.33 \mathrm{~d}-\mathrm{f}$ & $146.11 \mathrm{~d}$ & \multirow{3}{*}{$148.81 b$} \\
\hline & $75 \mathrm{mg} . \mathrm{L}^{-1}$ & $150.00 \mathrm{c}-\mathrm{e}$ & $148.00 \mathrm{~d}-\mathrm{f}$ & $150.33 \mathrm{c}-\mathrm{e}$ & $149.44 \mathrm{c}$ & \\
\hline & $150 \mathrm{mg} . \mathrm{L}^{-1}$ & $151.33 \mathrm{~cd}$ & $152.33 \mathrm{a}-\mathrm{d}$ & $149.00 \mathrm{~d}-\mathrm{f}$ & $150.89 \mathrm{c}$ & \\
\hline \multirow{3}{*}{$200 \mathrm{mg} \cdot \mathrm{L}^{-1}$} & $0 \mathrm{mg} \cdot \mathrm{L}^{-1}$ & $150.00 \mathrm{c}-\mathrm{e}$ & $147.67 d-f$ & $152.67 \mathrm{a}-\mathrm{d}$ & $150.11 b c$ & \multirow{3}{*}{$152.44 \mathrm{a}$} \\
\hline & $75 \mathrm{mg} . \mathrm{L}^{-1}$ & $155.00 \mathrm{a}-\mathrm{c}$ & $157.00 \mathrm{ab}$ & $147.67 d-f$ & $153.22 \mathrm{ab}$ & \\
\hline & $150 \mathrm{mg} . \mathrm{L}^{-1}$ & $152.67 \mathrm{a}-\mathrm{d}$ & $152.00 \mathrm{~b}-\mathrm{d}$ & $157.33 \mathrm{a}$ & $154.00 \mathrm{a}$ & \\
\hline \multicolumn{2}{|c|}{ Urtica Dioica } & $143.78 \mathrm{~b}$ & $144.78 \mathrm{ab}$ & $145.85 \mathrm{a}$ & \multirow{4}{*}{\multicolumn{2}{|c|}{ Disper root }} \\
\hline \multirow{3}{*}{$\begin{array}{c}\text { Prosopis } \\
\text { Farcta } \\
* \text { Urtica } \\
\text { Dioica }\end{array}$} & $0 \mathrm{mg} . \mathrm{L}^{-1}$ & $130.22 \mathrm{~d}$ & $133.44 \mathrm{c}$ & $135.78 \mathrm{c}$ & & \\
\hline & $100 \mathrm{mg} . \mathrm{L}^{-1}$ & $148.56 \mathrm{~b}$ & $148.67 \mathrm{~b}$ & $149.22 \mathrm{~b}$ & & \\
\hline & $200 \mathrm{mg} \cdot \mathrm{L}^{-1}$ & $152.56 \mathrm{a}$ & $152.22 \mathrm{a}$ & $152.56 \mathrm{a}$ & & \\
\hline \multirow{3}{*}{$\begin{array}{l}\text { Urtica } \\
\text { Dioica } \\
\text { *Disper } \\
\text { root }\end{array}$} & $0 \mathrm{mg} \cdot \mathrm{L}^{-1}$ & $138.56 \mathrm{e}$ & $139.67 \mathrm{e}$ & $142.89 \mathrm{~d}$ & $0 \mathrm{mg} \cdot \mathrm{L}^{-1}$ & $140.37 \mathrm{c}$ \\
\hline & $75 \mathrm{mg} . \mathrm{L}^{-1}$ & $144.89 \mathrm{~cd}$ & $147.00 \mathrm{a}-\mathrm{c}$ & $145.33 \mathrm{~b}-\mathrm{d}$ & $75 \mathrm{mg} . \mathrm{L}^{-1}$ & $145.74 b$ \\
\hline & $150 \mathrm{mg} . \mathrm{L}^{-1}$ & $147.88 \mathrm{ab}$ & $147.67 \mathrm{a}-\mathrm{c}$ & $149.33 \mathrm{a}$ & $150 \mathrm{mg} . \mathrm{L}^{-1}$ & $148.30 \mathrm{a}$ \\
\hline
\end{tabular}

Means within a row, column and their interactions, followed with the same letters are not significantly different from each other according to Duncan's multiple ranges test at significant level of 5\%. 


\section{4- Chlorophyll (SPAD)}

It's clear from the table 4 that spraying pomegranate transplant with Prosopis Farcta at 200 mg.L-1 chlorophyll present in the leaves significantly increased compared to the control. Chlorophyll content was also raised by spraying Urtica Dioica at both concentrations, most chlorophyll content took with spraying $200 \mathrm{mg} . \mathrm{L}-1$ compared to lowest value 35.16 at control. Same table showed that spraying pomegranate with Disper root at $150 \mathrm{mg} . \mathrm{L}-1$ increased significantly chlorophyll in the leaves compared to no spray. From Table 4 indicated that interactions of Prosopis Farcta and Urtica Dioica were significantly affected chlorophyll content in the leaf. The maximum values 44.82 were with interactions of spraying 200
mg.L-1 Prosopis Farcta and 100 mg.L-1 Urtica Dioica. Regarding the interaction of Prosopis Farcta + Disper root maximum value 45.51 was resulted from the interaction of spraying $200 \mathrm{mg}$.L-1 Prosopis Farcta +150 mg.L-1 Disper root took the maximum value. This table showed us the chlorophyll content in the leaves of transplants significantly increased especially at interactions between 200 mg.L-1 Urtica Dioica and 150 mg.L-1 Disper root gave the maximum value 40.11; In the interaction of the three factors, the mixture of Prosopis Farcta + Urtica Dioica + Disper root at 200 mg.L-1 + 200 mg.L-1 + 150 mg.L-1 respectively gave the highest value 48.97 compared to lowest value 28.33 from the interaction of 0 mg.L-1 Prosopis Farcta +0 mg.L-1 Urtica Dioica +0 mg.L1 Disper root.

Table 4. Effect of Prosopis Farcta extract, Urtica Dioica and Disper root and their interactions on chlorophyll (SPAD) of cv. Shahreban pomegranate transplant

\begin{tabular}{|c|c|c|c|c|c|c|}
\hline $\begin{array}{l}\text { Prosopis } \\
\text { Farcta }\end{array}$ & Disper root & $\begin{array}{c}\text { Urtica } \\
\text { Dioica } \\
\text { 0 mg.L }{ }^{-1} \\
\end{array}$ & $\begin{array}{c}\text { Urtica } \\
\text { Dioica } \\
100 \text { mg.L }^{-1} \\
\end{array}$ & $\begin{array}{c}\text { Urtica } \\
\text { Dioica } \\
200 \text { mg.L }^{-1} \\
\end{array}$ & $\begin{array}{c}\text { P.farcta } \\
* \text { Disper } \\
\text { root } \\
\end{array}$ & $\begin{array}{l}\text { Prosopis } \\
\text { farcta }\end{array}$ \\
\hline \multirow{3}{*}{$0 \mathrm{mg} \cdot \mathrm{L}^{-1}$} & $0 \mathrm{mg} . \mathrm{L}^{-1}$ & $28.23 \mathrm{~h}$ & $29.17 \mathrm{~h}$ & $29.30 \mathrm{gh}$ & $28.90 \mathrm{f}$ & \multirow{3}{*}{$30.69 \mathrm{c}$} \\
\hline & $75 \mathrm{mg} . \mathrm{L}^{-1}$ & $29.47 \mathrm{gh}$ & $31.07 f-h$ & $31.43 \mathrm{f}-\mathrm{h}$ & $30.66 \mathrm{ef}$ & \\
\hline & $150 \mathrm{mg} . \mathrm{L}^{-1}$ & $33.57 \mathrm{f}$ & $31.90 f-h$ & $32.10 \mathrm{f}-\mathrm{h}$ & $32.52 \mathrm{e}$ & \\
\hline \multirow{3}{*}{$100 \mathrm{mg} . \mathrm{L}^{-1}$} & $0 \mathrm{mg} . \mathrm{L}^{-1}$ & $30.50 f-h$ & $31.30 \mathrm{f}-\mathrm{h}$ & $30.43 \mathrm{f}-\mathrm{h}$ & $30.74 \mathrm{ef}$ & \multirow{3}{*}{$35.43 \mathrm{~b}$} \\
\hline & $75 \mathrm{mg} . \mathrm{L}^{-1}$ & $33.10 \mathrm{fg}$ & $37.40 \mathrm{e}$ & 39.37de & $36.62 \mathrm{~d}$ & \\
\hline & 150 mg. $\mathrm{L}^{-1}$ & 39.50de & $38.03 \mathrm{de}$ & $39.27 \mathrm{de}$ & $38.93 \mathrm{c}$ & \\
\hline \multirow{3}{*}{$200 \mathrm{mg} \cdot \mathrm{L}^{-1}$} & $0 \mathrm{mg} \cdot \mathrm{L}^{-1}$ & $41.00 \mathrm{c}-\mathrm{e}$ & $43.23 b c$ & $43.57 \mathrm{bc}$ & $42.60 \mathrm{~b}$ & \multirow{3}{*}{$43.36 \mathrm{a}$} \\
\hline & $75 \mathrm{mg} . \mathrm{L}^{-1}$ & $40.00 \mathrm{c}-\mathrm{e}$ & $44.93 \mathrm{~b}$ & $41.00 \mathrm{c}-\mathrm{e}$ & $41.98 \mathrm{~b}$ & \\
\hline & $150 \mathrm{mg} . \mathrm{L}^{-1}$ & $41.27 \mathrm{~cd}$ & $46.30 \mathrm{ab}$ & $48.97 \mathrm{a}$ & $45.51 \mathrm{a}$ & \\
\hline \multicolumn{2}{|c|}{ Urtica Dioica } & $35.16 \mathrm{~b}$ & $37.04 \mathrm{a}$ & $37.27 \mathrm{a}$ & \multirow{4}{*}{\multicolumn{2}{|c|}{ Disper root }} \\
\hline \multirow{3}{*}{$\begin{array}{c}\text { Prosopis } \\
\text { Farcta } \\
* \text { Urtica } \\
\text { Dioica } \\
\end{array}$} & 0 mg.L ${ }^{-1}$ & $30.42 \mathrm{~d}$ & $30.71 \mathrm{~d}$ & $30.94 \mathrm{~d}$ & & \\
\hline & $100 \mathrm{mg} . \mathrm{L}^{-1}$ & $34.37 \mathrm{c}$ & $35.58 \mathrm{c}$ & $36.36 \mathrm{c}$ & & \\
\hline & $200 \mathrm{mg} \cdot \mathrm{L}^{-1}$ & $40.76 \mathrm{~b}$ & $44.82 \mathrm{a}$ & $44.51 \mathrm{a}$ & & \\
\hline \multirow{3}{*}{$\begin{array}{l}\text { Urtica Dioica } \\
\text { *Disper root }\end{array}$} & $0 \mathrm{mg} \cdot \mathrm{L}^{-1}$ & $33.24 \mathrm{c}$ & $34.57 \mathrm{c}$ & $34.43 \mathrm{c}$ & $0 \mathrm{mg} \cdot \mathrm{L}^{-1}$ & $\begin{array}{c}34.08 \\
\mathrm{c}\end{array}$ \\
\hline & $75 \mathrm{mg} \cdot \mathrm{L}^{-1}$ & $34.19 \mathrm{c}$ & $37.80 \mathrm{~b}$ & $37.27 \mathrm{~b}$ & $75 \mathrm{mg} . \mathrm{L}^{-1}$ & $\begin{array}{c}36.42 \\
\mathrm{~b}\end{array}$ \\
\hline & $150 \mathrm{mg} . \mathrm{L}^{-1}$ & $38.11 \mathrm{ab}$ & $38.74 \mathrm{ab}$ & $40.11 \mathrm{a}$ & $150 \mathrm{mg} . \mathrm{L}^{-}$ & $\begin{array}{c}38.99 \\
a\end{array}$ \\
\hline
\end{tabular}

Means within a row, column and their interactions, followed with the same letters are not significantly different from each other according to Duncan's multiple ranges test at significant level of 5\%.

\section{5- $\quad$ Roots Number (Roots . Transplant -1)}

The roots number (roots. transplant -1) significantly increased by spraying $200 \mathrm{mg} . \mathrm{L}-1$ Prosopis Farcta at 8.56 (roots. transplant -1) which gave the highest value compare to no spraying 5.15 (roots. transplant -1). In this table we can see spraying of Urtica Dioica and Disper root gave the highest value of roots number (roots. transplant -1 ) compared no spraying. Table 5 indicates that the spraying of Prosopis Farcta with Urtica Dioica at both concentrations $200 \mathrm{mg}$.L- 1 had significantly effect on the roots number (roots. transplant -1) compare to control. Results from table 5 indicate the significant effect of interaction between spraying of Prosopis Farcta and Disper root on the roots number (roots. transplant -1), as results of the interaction between Prosopis Farcta and Disper root 
which recorded the highest the roots number (roots. transplant -1). We can found from the study interaction of Urtica Dioica and Disper root had the highest value at 8.33 (roots. transplant -1) compared control gave the lowest value 5.33 (roots. transplant -1). Table 5 also shows that the interaction among
Prosopis Farcta, Urtica Dioica and Disper root, had significant effect on the roots number (roots. transplant -1) of pomegranate transplant. The highest value was 10.00 (roots. transplant -1), and the lowest value 2.67 (roots. transplant -1 ).

Table 5. Effect of Prosopis Farcta extract, Urtica Dioica and Disper root and their interactions on roots number (roots. transplant -1) of cv. Shahreban pomegranate transplant.

\begin{tabular}{|c|c|c|c|c|c|c|}
\hline Prosopis Farcta & Disper root & $\begin{array}{c}\text { Urtica } \\
\text { Dioica } \\
0 \text { mg.L } \\
\end{array}$ & $\begin{array}{c}\text { Urtica } \\
\text { Dioica } \\
100 \text { mg.L }^{-1}\end{array}$ & $\begin{array}{c}\text { Urtica } \\
\text { Dioica } \\
200 \text { mg.L }^{-1}\end{array}$ & $\begin{array}{c}\text { P.farcta } * \\
\text { Disper } \\
\text { root }\end{array}$ & $\begin{array}{l}\text { Prosopis } \\
\text { farcta }\end{array}$ \\
\hline \multirow{3}{*}{$0 \mathrm{mg} . \mathrm{L}^{-1}$} & $0 \mathrm{mg} . \mathrm{L}^{-1}$ & $2.67 \mathrm{j}$ & $3.33 \mathrm{ij}$ & $6.00 \mathrm{e}-\mathrm{h}$ & $4.00 \mathrm{~d}$ & \multirow{3}{*}{$5.15 \mathrm{c}$} \\
\hline & $75 \mathrm{mg} . \mathrm{L}^{-1}$ & $4.67 \mathrm{hi}$ & $5.00 \mathrm{~g}-\mathrm{i}$ & $5.00 \mathrm{~g}-\mathrm{i}$ & $4.89 \mathrm{~d}$ & \\
\hline & 150 mg.L - $^{-1}$ & $6.00 \mathrm{e}-\mathrm{h}$ & $7.00 \mathrm{~d}-\mathrm{g}$ & $6.67 \mathrm{~d}-\mathrm{h}$ & $6.56 \mathrm{c}$ & \\
\hline \multirow{3}{*}{$100 \mathrm{mg} \cdot \mathrm{L}^{-1}$} & $0 \mathrm{mg} . \mathrm{L}^{-1}$ & $6.33 d-h$ & $6.33 \mathrm{~d}-\mathrm{h}$ & $6.33 \mathrm{~d}-\mathrm{h}$ & $6.33 \mathrm{c}$ & \multirow{3}{*}{$6.63 \mathrm{~b}$} \\
\hline & $75 \mathrm{mg} . \mathrm{L}^{-1}$ & $6.33 \mathrm{~d}-\mathrm{h}$ & $7.00 \mathrm{~d}-\mathrm{g}$ & $6.00 \mathrm{e}-\mathrm{h}$ & $6.44 \mathrm{c}$ & \\
\hline & 150 mg.L . $^{-1}$ & $5.67 \mathrm{f}-\mathrm{h}$ & $7.33 c-f$ & $8.33 \mathrm{a}-\mathrm{d}$ & $7.11 b c$ & \\
\hline \multirow{3}{*}{$200 \mathrm{mg} . \mathrm{L}^{-1}$} & $0 \mathrm{mg} . \mathrm{L}^{-1}$ & $7.00 \mathrm{~d}-\mathrm{g}$ & $8.00 \mathrm{~b}-\mathrm{e}$ & $8.00 \mathrm{~b}-\mathrm{e}$ & $7.67 \mathrm{~b}$ & \multirow{3}{*}{$8.56 \mathrm{a}$} \\
\hline & $75 \mathrm{mg} . \mathrm{L}^{-1}$ & $7.33 c-f$ & $9.00 \mathrm{a}-\mathrm{c}$ & $9.67 \mathrm{ab}$ & $8.67 \mathrm{a}$ & \\
\hline & $150 \mathrm{mg} . \mathrm{L}^{-1}$ & $9.00 \mathrm{a}-\mathrm{c}$ & $9.00 \mathrm{a}-\mathrm{c}$ & $10.00 \mathrm{a}$ & $9.33 \mathrm{a}$ & \\
\hline \multicolumn{2}{|c|}{ Urtica Dioica } & $6.11 \mathrm{~b}$ & $6.89 \mathrm{a}$ & $7.33 \mathrm{a}$ & \multirow{4}{*}{\multicolumn{2}{|c|}{ Disper root }} \\
\hline \multirow{3}{*}{$\begin{array}{l}\text { Prosopis Farcta } \\
* \text { Urtica Dioica }\end{array}$} & $0 \mathrm{mg} . \mathrm{L}^{-1}$ & $4.44 \mathrm{f}$ & $5.11 \mathrm{ef}$ & $5.89 \mathrm{de}$ & & \\
\hline & 100 mg.L . $^{-1}$ & $6.11 \mathrm{de}$ & $6.89 \mathrm{~cd}$ & $6.89 \mathrm{~cd}$ & & \\
\hline & $200 \mathrm{mg} . \mathrm{L}^{-1}$ & $7.78 \mathrm{bc}$ & $8.67 \mathrm{ab}$ & $9.22 \mathrm{a}$ & & \\
\hline \multirow{3}{*}{$\begin{array}{l}\text { Urtica Dioica } \\
* \text { Disper root }\end{array}$} & $0 \mathrm{mg} . \mathrm{L}^{-1}$ & $5.33 \mathrm{e}$ & $5.89 \mathrm{de}$ & $6.78 \mathrm{~b}-\mathrm{d}$ & $0 \mathrm{mg} . \mathrm{L}^{-1}$ & $6.00 \mathrm{c}$ \\
\hline & $75 \mathrm{mg} . \mathrm{L}^{-1}$ & $6.11 \mathrm{c}-\mathrm{e}$ & $7.00 \mathrm{bc}$ & $6.89 \mathrm{~b}-\mathrm{d}$ & $75 \mathrm{mg} . \mathrm{L}^{-1}$ & $6.67 \mathrm{~b}$ \\
\hline & $150 \mathrm{mg.L^{-1 }}$ & $6.89 \mathrm{~b}-\mathrm{d}$ & $7.78 \mathrm{ab}$ & $8.33 \mathrm{a}$ & $150 \mathrm{mg} . \mathrm{L}^{-1}$ & $7.67 \mathrm{a}$ \\
\hline
\end{tabular}

Means within a row, column and their interactions, followed with the same letters are not significantly different from each other according to Duncan's multiple ranges test at significant level of 5\%.

\section{6- $\quad$ Root Length (cm)}

Table 6 indicated that the spraying of Prosopis Farcta with $200 \mathrm{mg} . \mathrm{L}-1$ have a significant effect on root length at $40.57 \mathrm{~cm}$ gave the highest value. The spraying of Urtica Dioica on pomegranate seedling no significantly affected on root length when the process did. It's obvious from this table spraying of Disper root at $150 \mathrm{mg} . \mathrm{L}-1$ concentration had significant effect on root length compared to control. Regarding the combination between Prosopis Farcta and Urtica Dioica table 6 shows that the interaction of both had the highest value of root length $41.63 \mathrm{~cm}$ compare to control 28.79. Table 6 also indicated that the interaction of Prosopis Farcta and Disper root on root length gave the highest value in the interaction at spraying $200 \mathrm{mg}$.L-1 Prosopis Farcta and 75 mg.L-1 Disper root. Regarding the combination between Urtica Dioica and Disper root in this table shows that this interaction not affected on root length of pomegranate transplants. Same table have the mixture of the three factors, the most value was showed from the interaction of $200 \mathrm{mg}$.L-1 Prosopis Farcta + 200 mg.L-1 Urtica Dioica +0 mg.L-1 Disper root compared with the minimum value from control.

Table 6. Effect of Prosopis Farcta extract, Urtica Dioica and Disper root and their interactions on root length (cm) of cv. Shahreban pomegranate transplant.

\begin{tabular}{|c|c|c|c|c|c|c|}
\hline $\begin{array}{c}\text { Prosopis } \\
\text { Farcta }\end{array}$ & Disper root & $\begin{array}{c}\text { Urtica } \\
\text { Dioica } \\
\text { 0 mg.L }{ }^{-1}\end{array}$ & $\begin{array}{c}\text { Urtica } \\
\text { Dioica } \\
100 \text { mg.L }^{-1}\end{array}$ & $\begin{array}{c}\text { Urtica } \\
\text { Dioica } \\
200 \text { mg.L }^{-1}\end{array}$ & $\begin{array}{l}\text { P.farcta } \\
* \text { Disper } \\
\text { root }\end{array}$ & $\begin{array}{c}\text { Prosopis } \\
\text { farcta }\end{array}$ \\
\hline \multirow{3}{*}{$0 \mathrm{mg} \cdot \mathrm{L}^{-1}$} & $0 \mathrm{mg} . \mathrm{L}^{-1}$ & $25.80 \mathrm{~g}$ & $26.73 \mathrm{fg}$ & $26.50 \mathrm{fg}$ & $26.34 \mathrm{~d}$ & \multirow{3}{*}{$28.83 \mathrm{c}$} \\
\hline & $75 \mathrm{mg} . \mathrm{L}^{-1}$ & $30.03 \mathrm{fg}$ & $29.90 f g$ & $29.47 \mathrm{fg}$ & $29.80 \mathrm{c}$ & \\
\hline & $150 \mathrm{mg} . \mathrm{L}^{-1}$ & $30.53 \mathrm{e}-\mathrm{g}$ & $29.43 \mathrm{fg}$ & $31.10 \mathrm{~d}-\mathrm{g}$ & $30.36 \mathrm{c}$ & \\
\hline 100 mg.L ${ }^{-1}$ & $0 \mathrm{mg} . \mathrm{L}^{-1}$ & $30.13 \mathrm{fg}$ & $35.80 \mathrm{~b}-\mathrm{e}$ & $30.67 \mathrm{e}-\mathrm{g}$ & $32.20 \mathrm{c}$ & $33.58 \mathrm{~b}$ \\
\hline
\end{tabular}




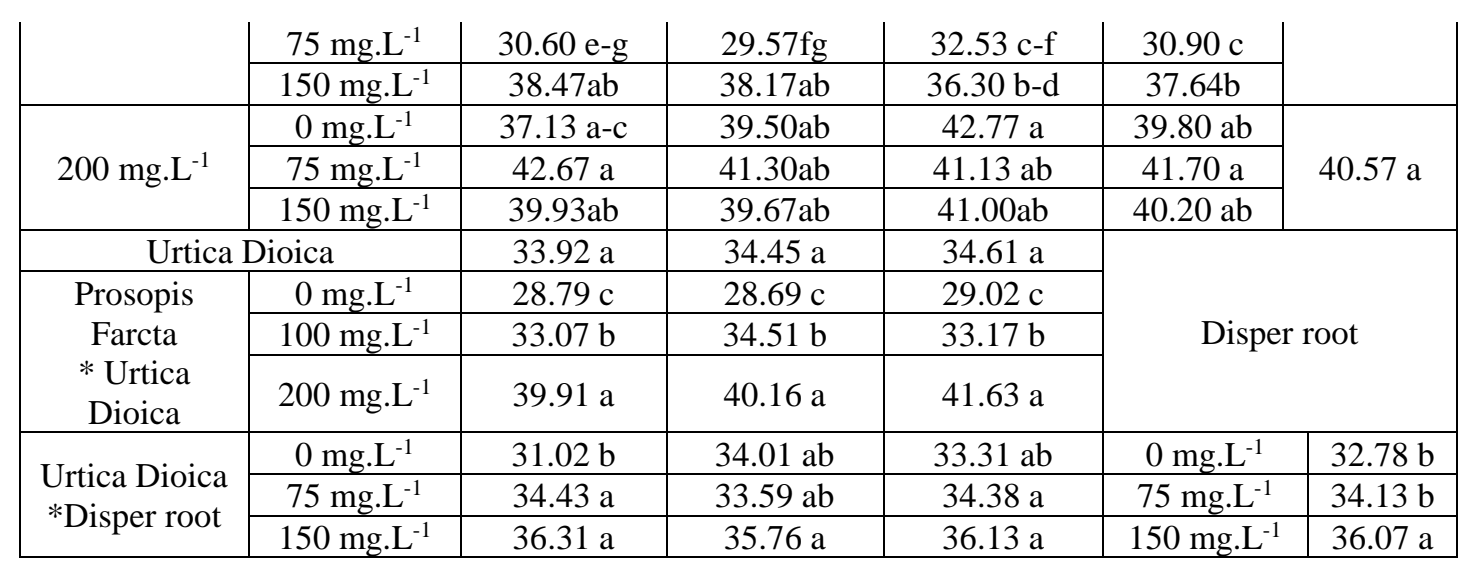

Means within a row, column and their interactions, followed with the same letters are not significantly different from each other according to Duncan's multiple ranges test at significant level of 5\%.

\section{7- $\quad$ Leaf Nitrogen Content (\%)}

In the table 7 obviously indicated that the foliar application of Prosopis Farcta about nitrogen percentage in leaf of the pomegranate transplant had significantly effected. The same table indicates that spraying of $200 \mathrm{mg} . \mathrm{L}-1$ Urtica Dioica and 150 mg.L-1 Disper root significantly affected on the nitrogen percentage compared to no spray gave the lowest value. Data in the table 7 shows that the interactions among Prosopis Farcta and Urtica Dioica significantly affected on nitrogen percentage in leaf of pomegranate, results showed that highest nitrogen $2.108 \%$ was registered from the combination of spraying 200 mg.L-1 Prosopis Farcta + 200 mg.L-1 Urtica Dioica, but the minimum value $1.661 \%$ was recorded at control. For the interactions between Prosopis Farcta and
Disper root, the results indicated that spraying of 200 mg.L-1 Prosopis Farcta + 150 mg.L-1 Disper root had the maximum significant nitrogen percentage $2.144 \%$ and $1.639 \%$ it was minimum value which recorded from control. The percentage of nitrogen in leaf of pomegranate transplant was affected by the interactions of Urtica Dioica and Disper root, table 7 pointed that the maximum value was took from 200 mg.L-1 Urtica Dioica + $150 \mathrm{mg}$.L-1 Disper root, but the lowest value was registered from non-spray . Regarding the interactions among the three studied factors, the same table illustrates that highest nitrogen percentage in leaf of pomegranate seedlings 2.507 $\%$ was recorded from combination of $200 \mathrm{mg} . \mathrm{L}-1$ Prosopis Farcta +200 mg.L-1 Urtica Dioica + 150 mg.L-1 Disper root, which significantly differed from other interactions especially the interactions of control.

Table 7. Effect of Prosopis Farcta extract, Urtica Dioica and Disper root and their interactions on leaf nitrogen content (\%) of cv. Shahreban pomegranate transplant

\begin{tabular}{|c|c|c|c|c|c|c|}
\hline $\begin{array}{c}\text { Prosopis } \\
\text { Farcta }\end{array}$ & Disper root & $\begin{array}{c}\text { Urtica } \\
\text { Dioica } \\
\text { 0 mg.L }{ }^{-1} \\
\end{array}$ & $\begin{array}{c}\text { Urtica } \\
\text { Dioica } \\
100 \text { mg.L }{ }^{-1}\end{array}$ & $\begin{array}{c}\text { Urtica } \\
\text { Dioica } \\
200 \text { mg.L }{ }^{-1} \\
\end{array}$ & $\begin{array}{c}\text { P.farcta } \\
\text { * Disper } \\
\text { root }\end{array}$ & $\begin{array}{l}\text { Prosopis } \\
\text { farcta }\end{array}$ \\
\hline \multirow{3}{*}{$0 \mathrm{mg} . \mathrm{L}^{-1}$} & $0 \mathrm{mg} . \mathrm{L}^{-1}$ & $1.637 \mathrm{~h}$ & $1.640 \mathrm{~h}$ & $1.640 \mathrm{~h}$ & $1.639 \mathrm{f}$ & \multirow{3}{*}{$1.663 \mathrm{c}$} \\
\hline & $75 \mathrm{mg} . \mathrm{L}^{-1}$ & $1.660 \mathrm{~h}$ & $1.657 \mathrm{~h}$ & $1.703 \mathrm{f}-\mathrm{h}$ & $1.673 \mathrm{ef}$ & \\
\hline & $150 \mathrm{mg} . \mathrm{L}^{-1}$ & $1.687 \mathrm{gh}$ & $1.663 \mathrm{~h}$ & $1.680 \mathrm{gh}$ & $1.677 \mathrm{ef}$ & \\
\hline \multirow{3}{*}{$100 \mathrm{mg} \cdot \mathrm{L}^{-1}$} & $0 \mathrm{mg} . \mathrm{L}^{-1}$ & $1.687 \mathrm{gh}$ & $1.700 \mathrm{f}-\mathrm{h}$ & $1.727 \mathrm{e}-\mathrm{h}$ & $1.704 \mathrm{ef}$ & \multirow{3}{*}{$1.749 \mathrm{~b}$} \\
\hline & $75 \mathrm{mg} . \mathrm{L}^{-1}$ & $1.713 \mathrm{f}-\mathrm{h}$ & $1.737 \mathrm{e}-\mathrm{h}$ & $1.783 \mathrm{~d}-\mathrm{h}$ & $1.744 \mathrm{de}$ & \\
\hline & $150 \mathrm{mg} . \mathrm{L}^{-1}$ & $1.787 \mathrm{~d}-\mathrm{h}$ & $1.783 \mathrm{~d}-\mathrm{h}$ & $1.820 \mathrm{c}-\mathrm{g}$ & $1.797 \mathrm{~cd}$ & \\
\hline \multirow{3}{*}{$200 \mathrm{mg} \cdot \mathrm{L}^{-1}$} & $0 \mathrm{mg} . \mathrm{L}^{-1}$ & $1.830 \mathrm{c}-\mathrm{g}$ & $1.873 \mathrm{~b}-\mathrm{e}$ & $1.850 \mathrm{c}-\mathrm{f}$ & $1.851 \mathrm{bc}$ & \multirow{3}{*}{$1.973 \mathrm{a}$} \\
\hline & 75 mg. $\mathrm{L}^{-1}$ & $1.893 \mathrm{~b}-\mathrm{d}$ & $1.907 \mathrm{~b}-\mathrm{d}$ & $1.967 \mathrm{bc}$ & $1.922 \mathrm{~b}$ & \\
\hline & $150 \mathrm{mg} . \mathrm{L}^{-1}$ & $1.923 \mathrm{~b}-\mathrm{d}$ & $2.003 \mathrm{~b}$ & $2.507 \mathrm{a}$ & $2.144 \mathrm{a}$ & \\
\hline \multicolumn{2}{|c|}{ Urtica Dioica } & $1.757 \mathrm{~b}$ & $1.774 \mathrm{~b}$ & $1.853 \mathrm{a}$ & \multirow{4}{*}{\multicolumn{2}{|c|}{ Disper root }} \\
\hline \multirow{3}{*}{$\begin{array}{l}\text { Prosopis } \\
\text { Farcta }\end{array}$} & $0 \mathrm{mg} . \mathrm{L}^{-1}$ & $1.661 \mathrm{e}$ & $1.653 \mathrm{de}$ & $1.674 \mathrm{de}$ & & \\
\hline & $100 \mathrm{mg} \cdot \mathrm{L}^{-1}$ & $1.729 \mathrm{c}-\mathrm{e}$ & $1.740 \mathrm{~cd}$ & $1.777 \mathrm{c}$ & & \\
\hline & $200 \mathrm{mg} \cdot \mathrm{L}^{-1}$ & $1.882 \mathrm{~b}$ & $1.928 \mathrm{~b}$ & $2.108 \mathrm{a}$ & & \\
\hline
\end{tabular}




\begin{tabular}{|c|c|c|c|c|c|c|}
\hline $\begin{array}{c}* \text { Urtica } \\
\text { Dioica }\end{array}$ & & & & & & \\
\hline \multirow{3}{*}{$\begin{array}{c}\text { Urtica } \\
\text { Dioica } \\
\text { *Disper } \\
\text { root }\end{array}$} & $0 \mathrm{mg} . \mathrm{L}^{-1}$ & $1.718 \mathrm{c}$ & $1.738 \mathrm{bc}$ & $1.739 b c$ & $0 \mathrm{mg} \cdot \mathrm{L}^{-1}$ & $1.731 \mathrm{c}$ \\
\hline & $75 \mathrm{mg} \cdot \mathrm{L}^{-1}$ & $1.756 b c$ & $1.767 b c$ & $1.818 \mathrm{~b}$ & $75 \mathrm{mg} . \mathrm{L}^{-1}$ & $1.780 \mathrm{~b}$ \\
\hline & $150 \mathrm{mg} \cdot \mathrm{L}^{-1}$ & $1.799 b c$ & $1.817 \mathrm{~b}$ & $2.002 \mathrm{a}$ & $150 \mathrm{mg} . \mathrm{L}^{-1}$ & $1.873 \mathrm{a}$ \\
\hline
\end{tabular}

Means within a row, column and their interactions, followed with the same letters are not significantly different from each other according to Duncan's multiple ranges test at significant level of 5\%.

\section{8- $\quad$ Leaf Phosphorus Content (\%)}

Table 8 points out that spraying of Prosopis Farcta on pomegranate transplant had significant effect on phosphorus percentage concentration in leaf of Shehreban cultivar. Foliar application of Urtica Dioica showed concentration with 200 mg.L-1 increased phosphorus percentage in leaf significantly compared to no spray. Data in same table shows that the spraying of Disper root significantly affected on percentage of phosphorus in leaf the most value was $0.201 \%$. Table 8 shows that the interactions of Prosopis Farcta + Urtica Dioica significantly effected on percentage of phosphorus in the leaf, results pointed that the highest phosphorus percentage $0.46 \%$ compared to the lowest value was registered at control $0.126 \%$.
The interactions between Prosopis Farcta and the phosphorus percentage significantly increased by spraying Disper root, the maximum value was $0.258 \%$ was recorded from combination of spray 200 mg.L-1 Prosopis Farcta and 150 mg.L-1 Disper root as compared to other interactions treatments. Table 8 also showed the combination among Urtica Dioica and Disper root. This combination increased phosphorus percentage in form significant in leaf of pomegranate transplant compare to control. Concerning the interactions of research factors, results indicated that the highest phosphorus $0.67 \%$ recorded from the interactions of $200 \mathrm{mg} . \mathrm{L}-1$ Prosopis Farcta $+200 \mathrm{mg}$.L-1 Urtica Dioica and 150 mg.L-1 Disper root compared to the interactions 0 mg.L-1 Prosopis Farcta +0 mg.L-1 Urtica Dioica and $0 \mathrm{mg}$.L-1 Disper root that recorded the lowest value $0.113 \%$.

Table 8. Effect of Prosopis Farcta extract, Urtica Dioica and Disper root and their interactions on leaf phosphorus content (\%) of cv. Shahreban pomegranate transplant.

\begin{tabular}{|c|c|c|c|c|c|c|}
\hline $\begin{array}{l}\text { Prosopis } \\
\text { Farcta }\end{array}$ & Disper root & $\begin{array}{c}\text { Urtica } \\
\text { Dioica } \\
\text { 0 mg.L }{ }^{-1}\end{array}$ & $\begin{array}{c}\text { Urtica } \\
\text { Dioica } \\
100 \text { mg.L }{ }^{-1}\end{array}$ & $\begin{array}{c}\text { Urtica } \\
\text { Dioica } \\
200 \text { mg.L }{ }^{-1}\end{array}$ & $\begin{array}{c}\text { P.farcta } \\
* \text { Disper } \\
\text { root }\end{array}$ & $\begin{array}{c}\text { Prosopis } \\
\text { farcta }\end{array}$ \\
\hline \multirow{3}{*}{$0 \mathrm{mg} \cdot \mathrm{L}^{-1}$} & $0 \mathrm{mg} \cdot \mathrm{L}^{-1}$ & $0.113 \mathrm{j}$ & $0.143 \mathrm{~g}-\mathrm{j}$ & $0.130 \mathrm{~h}-\mathrm{j}$ & $0.129 \mathrm{~g}$ & \multirow{3}{*}{$0.140 \mathrm{c}$} \\
\hline & $75 \mathrm{mg} \cdot \mathrm{L}^{-1}$ & $0.123 \mathrm{ij}$ & $0.140 \mathrm{~g}-\mathrm{j}$ & $0.157 \mathrm{f}-\mathrm{i}$ & $0.140 \mathrm{fg}$ & \\
\hline & $150 \mathrm{mg} \cdot \mathrm{L}^{-1}$ & $0.140 \mathrm{~g}-\mathrm{j}$ & $0.160 \mathrm{f}-\mathrm{h}$ & $0.150 \mathrm{~g}-\mathrm{i}$ & $0.150 \mathrm{ef}$ & \\
\hline \multirow{3}{*}{$100 \mathrm{mg} . \mathrm{L}^{-1}$} & $0 \mathrm{mg} . \mathrm{L}^{-1}$ & $0.147 \mathrm{~g}-\mathrm{j}$ & $0.170 \mathrm{fg}$ & $0.207 \mathrm{c}-\mathrm{e}$ & $0.174 \mathrm{~d}$ & \multirow{3}{*}{$0.179 \mathrm{~b}$} \\
\hline & $75 \mathrm{mg} \cdot \mathrm{L}^{-1}$ & $0.150 \mathrm{~g}-\mathrm{i}$ & $0.167 \mathrm{fg}$ & $0.177 \mathrm{e}-\mathrm{g}$ & $0.164 \mathrm{de}$ & \\
\hline & $150 \mathrm{mg} \cdot \mathrm{L}^{-1}$ & $0.177 \mathrm{e}-\mathrm{g}$ & $0.190 \mathrm{~d}-\mathrm{f}$ & $0.223 \mathrm{~b}-\mathrm{d}$ & $0.197 \mathrm{c}$ & \\
\hline \multirow{3}{*}{$200 \mathrm{mg} \cdot \mathrm{L}^{-1}$} & $0 \mathrm{mg} \cdot \mathrm{L}^{-1}$ & $0.243 \mathrm{ab}$ & $0.227 b c$ & $0.220 \mathrm{~b}-\mathrm{d}$ & $0.230 \mathrm{~b}$ & \multirow{3}{*}{$0.240 \mathrm{a}$} \\
\hline & $75 \mathrm{mg} \cdot \mathrm{L}^{-1}$ & $0.240 \mathrm{a}-\mathrm{c}$ & $0.220 \mathrm{c}-\mathrm{e}$ & $0.240 \mathrm{a}-\mathrm{c}$ & $0.233 \mathrm{~b}$ & \\
\hline & $150 \mathrm{mg} \cdot \mathrm{L}^{-1}$ & $0.253 \mathrm{ab}$ & $0.253 \mathrm{ab}$ & $0.267 \mathrm{a}$ & $0.258 \mathrm{a}$ & \\
\hline \multicolumn{2}{|c|}{ Urtica Dioica } & $0.176 \mathrm{~b}$ & $0.186 \mathrm{~b}$ & $0.197 \mathrm{a}$ & \multirow{4}{*}{\multicolumn{2}{|c|}{ Disper root }} \\
\hline \multirow{3}{*}{$\begin{array}{c}\text { Prosopis } \\
\text { Farcta } \\
* \text { Urtica } \\
\text { Dioica } \\
\end{array}$} & $0 \mathrm{mg} \cdot \mathrm{L}^{-1}$ & $0.126 \mathrm{e}$ & $0.148 \mathrm{~d}$ & $0.146 \mathrm{~d}$ & & \\
\hline & $100 \mathrm{mg} . \mathrm{L}^{-1}$ & $0.158 \mathrm{~cd}$ & $0.176 \mathrm{c}$ & $0.202 \mathrm{~b}$ & & \\
\hline & $200 \mathrm{mg} \cdot \mathrm{L}^{-1}$ & $0.246 \mathrm{a}$ & $0.233 \mathrm{a}$ & $0.242 \mathrm{a}$ & & \\
\hline \multirow{3}{*}{$\begin{array}{c}\text { Urtica } \\
\text { Dioica } \\
\text { *Disper root }\end{array}$} & $0 \mathrm{mg} \cdot \mathrm{L}^{-1}$ & $0.168 \mathrm{~d}$ & $0.180 \mathrm{~cd}$ & $0.186 \mathrm{~b}-\mathrm{d}$ & $0 \mathrm{mg} . \mathrm{L}^{-1}$ & $0.178 \mathrm{~b}$ \\
\hline & $75 \mathrm{mg} . \mathrm{L}^{-1}$ & $0.171 \mathrm{~cd}$ & $0.176 \mathrm{~cd}$ & $0.191 \mathrm{bc}$ & $75 \mathrm{mg} . \mathrm{L}^{-1}$ & $0.179 \mathrm{~b}$ \\
\hline & $150 \mathrm{mg} \cdot \mathrm{L}^{-1}$ & $0.190 \mathrm{bc}$ & $0.201 \mathrm{ab}$ & $0.213 \mathrm{a}$ & $150 \mathrm{mg} . \mathrm{L}$ & $0.201 \mathrm{a}$ \\
\hline
\end{tabular}

Means within a row, column and their interactions, followed with the same letters are not significantly different from each other according to Duncan's multiple ranges test at significant level of 5\%.

\section{9- Leaf Potassium Content (\%)}

Table 9 showed that spraying of Prosopis Farcta had significance effect on potassium 
concentration in leaf of pomegranate transplant. Potassium percentage in leaf affected significantly by foliar application of Urtica Dioica, which records the maximum value $1.302 \%$ at the concentration $200 \mathrm{mg}$.L-1 compared to the control. Data in table 9 shows that spray of Disper root significantly affected on potassium percentage of leaves pomegranate. This application was the most valuable $1.324 \%$ while the control had the lowest value $1.252 \%$. For the interactions of both studied factors Prosopis Farcta and Urtica Dioica, data showed that the interactions between Prosopis Farcta + Urtica Dioica record significant impact on potassium concentration, and spraying pomegranate seedlings with $200 \mathrm{mg}$.L-1 Prosopis Farcta $+200 \mathrm{mg} . \mathrm{L}-1$ In comparison to the minimal value achieved at control, Urtica Dioica produced the highest potassium percentage in the leaf. The combination between Prosopis Farcta and Disper root had significant effect on potassium percentage in leaf, the maximum leaf potassium percentage was recorded as a result of the interactions between spray of 200 mg.L-1 Prosopis Farcta and 150 mg.L1 Disper root, but the lowest value was noticed from the interactions of $0 \mathrm{mg}$.L-1 Prosopis Farcta and 0 mg.L-1 Disper root. Table 9 shows that the interactions of Urtica Dioica + Disper root were significantly affected on potassium percentage in leaf of pomegranate, the results displayed that combination of 200 mg.L-1 Urtica Dioica +150 mg.L-1 Disper root gave the highest value and control took the lowest value of potassium percentage in leaf of pomegranate transplant $\mathrm{cv}$. shehreban. In the same table also demonstrated the combination of three factors were studied. Results registered that interactions of Prosopis Farcta + Urtica Dioica + Disper root significantly increased potassium percentage in leaf of pomegranate, the highest potassium percentage $1.433 \%$ was recorded from the interactions of 200 mg.L-1 Prosopis Farcta +200 mg.L-1 Urtica Dioica +150 mg.L-1 Disper root, which significantly surpass to lowest value $1.107 \%$ recorded in control.

Table 9. Effect of Prosopis Farcta extract, Urtica Dioica and Disper root and their interactions on leaf potassium

\begin{tabular}{|c|c|c|c|c|c|c|}
\hline $\begin{array}{l}\text { Prosopis } \\
\text { Farcta }\end{array}$ & Disper root & $\begin{array}{c}\text { Urtica } \\
\text { Dioica } \\
0 \text { mg.L }{ }^{-1} \\
\end{array}$ & $\begin{array}{c}\text { Urtica } \\
\text { Dioica } \\
100 \text { mg.L }^{-1}\end{array}$ & $\begin{array}{c}\text { Urtica } \\
\text { Dioica } \\
200 \text { mg.L }^{-1} \\
\end{array}$ & $\begin{array}{c}\text { P.farcta } \\
* \text { Disper } \\
\text { root } \\
\end{array}$ & $\begin{array}{c}\text { Prosopis } \\
\text { farcta }\end{array}$ \\
\hline \multirow{3}{*}{$0 \mathrm{mg} . \mathrm{L}^{-1}$} & $0 \mathrm{mg} . \mathrm{L}^{-1}$ & $1.107 \mathrm{~m}$ & $1.140 \mathrm{k}-\mathrm{m}$ & $1.127 \mathrm{~lm}$ & $1.124 \mathrm{~g}$ & \multirow{3}{*}{$1.153 \mathrm{c}$} \\
\hline & $75 \mathrm{mg} . \mathrm{L}^{-1}$ & $1.140 \mathrm{k}-\mathrm{m}$ & $1.160 \mathrm{kl}$ & $1.137 \mathrm{k}-\mathrm{m}$ & $1.146 \mathrm{f}$ & \\
\hline & $150 \mathrm{mg} . \mathrm{L}^{-1}$ & $1.153 \mathrm{kl}$ & $1.173 \mathrm{k}$ & $1.237 \mathrm{ij}$ & $1.188 \mathrm{e}$ & \\
\hline \multirow{3}{*}{$100 \mathrm{mg} . \mathrm{L}^{-1}$} & $0 \mathrm{mg} . \mathrm{L}^{-1}$ & $1.227 \mathrm{j}$ & $1.263 \mathrm{hi}$ & $1.270 \mathrm{hi}$ & $1.253 \mathrm{~d}$ & \multirow{3}{*}{$1.307 \mathrm{~b}$} \\
\hline & $75 \mathrm{mg} . \mathrm{L}^{-1}$ & $1.273 \mathrm{~h}$ & $1.320 \mathrm{~g}$ & $1.330 \mathrm{fg}$ & $1.308 \mathrm{c}$ & \\
\hline & $150 \mathrm{mg} \cdot \mathrm{L}^{-1}$ & $1.347 \mathrm{e}-\mathrm{g}$ & $1.360 \mathrm{~d}-\mathrm{f}$ & $1.377 \mathrm{c}-\mathrm{e}$ & $1.361 \mathrm{~b}$ & \\
\hline \multirow{3}{*}{$200 \mathrm{mg} . \mathrm{L}^{-1}$} & $0 \mathrm{mg} . \mathrm{L}^{-1}$ & $1.360 \mathrm{~d}-\mathrm{f}$ & $1.387 \mathrm{~b}-\mathrm{d}$ & $1.390 \mathrm{~b}-\mathrm{d}$ & $1.379 \mathrm{~b}$ & \multirow{3}{*}{$1.405 \mathrm{a}$} \\
\hline & $75 \mathrm{mg} . \mathrm{L}^{-1}$ & $1.407 \mathrm{a}-\mathrm{c}$ & $1.410 \mathrm{a}-\mathrm{c}$ & $1.420 \mathrm{ab}$ & $1.412 \mathrm{a}$ & \\
\hline & $150 \mathrm{mg} \cdot \mathrm{L}^{-1}$ & $1.420 \mathrm{ab}$ & $1.417 \mathrm{ab}$ & $1.433 \mathrm{a}$ & $1.423 \mathrm{a}$ & \\
\hline \multicolumn{2}{|c|}{ Urtica Dioica } & $1.270 \mathrm{~b}$ & $1.292 \mathrm{a}$ & $1.302 \mathrm{a}$ & \multirow{4}{*}{\multicolumn{2}{|c|}{ Disper root }} \\
\hline \multirow{3}{*}{$\begin{array}{c}\text { Prosopis } \\
\text { Farcta } \\
* \text { Urtica } \\
\text { Dioica }\end{array}$} & 0 mg. $\mathrm{L}^{-1}$ & $1.133 \mathrm{e}$ & $1.158 \mathrm{~d}$ & $1.167 \mathrm{~d}$ & & \\
\hline & $100 \mathrm{mg} . \mathrm{L}^{-1}$ & $1.282 \mathrm{c}$ & $1.314 \mathrm{~b}$ & $1.326 \mathrm{~b}$ & & \\
\hline & $200 \mathrm{mg} . \mathrm{L}^{-1}$ & $1.396 \mathrm{a}$ & $1.404 \mathrm{a}$ & $1.414 \mathrm{a}$ & & \\
\hline \multirow{3}{*}{$\begin{array}{l}\text { Urtica Dioica } \\
\text { *Disper root }\end{array}$} & $0 \mathrm{mg} \cdot \mathrm{L}^{-1}$ & $1.231 \mathrm{e}$ & $1.263 \mathrm{~d}$ & $1.262 \mathrm{~d}$ & $0 \mathrm{mg} . \mathrm{L}^{-1}$ & $\begin{array}{c}1.252 \\
\mathrm{c}\end{array}$ \\
\hline & $75 \mathrm{mg} . \mathrm{L}^{-1}$ & $1.273 \mathrm{~d}$ & $1.297 \mathrm{bc}$ & $1.296 \mathrm{c}$ & $75 \mathrm{mg} \cdot \mathrm{L}^{-1}$ & $\begin{array}{c}1.289 \\
b\end{array}$ \\
\hline & $150 \mathrm{mg} . \mathrm{L}^{-1}$ & $1.307 \mathrm{bc}$ & $1.317 \mathrm{~b}$ & $1.349 \mathrm{a}$ & $150 \mathrm{mg} . \mathrm{L}^{-}$ & $\begin{array}{c}1.324 \\
\mathrm{a}\end{array}$ \\
\hline
\end{tabular}

Means within a row, column and their interactions, followed with the same letters are not significantly different from each other according to Duncan's multiple ranges test at significant level of 5\%.

To the best of our knowledge, it's obvious from all tables during conversation, that spraying of Prosopis Farcta, Urtica Dioica and Disper root had a significantly effected on mostly all vegetative growth and nutrients contents of pomegranate 
transplant. The positive effect of Prosopis Farcta might be attributed to the source of protein, (Houda et al, 2015). Total proteins play an important role in transport of vitamins, hormones, enzymes (Arash and Ghazaghi, 2012). The significant effect of Urtica Dioica on transplants could be attributed to contain vitamins, calcium, iron and carbohydrates (Rutto et al. 2013). Disper Root significant effect on the growth of pomegranate transplants may be because of this product contains free amino acids, polysaccharides, vitamins, humic extract, phosphorus, calcium, potassium. (www.edenmodemadriculture.com Spain).

\section{Conclusion}

As indicated in the all data application of Prosopis Farcta, Urtica Dioica and Disper root contain sufficient amount of essential nutrient elements lead to prosper all studied parameters compared with control. Application of Prosopis Farcta, Urtica Dioica and Disper root have a significant effect on number of branches, transplant height, number of leaves, chlorophyll, number of roots, root length, leaf nitrogen content, leaf phosphorus content, leaf potassium content. The combination of three studied factors had a significantly surpass in improving all parameters studied in this experiment compared with in control.

\section{Conflict of Interest}

The authors state that there were no commercial or financial relationships that may be considered as a potential conflict of interest during the research.

\section{Acknowledgments}

Appreciations and endless thanks to the Department of Horticulture at the College of Agricultural Engineering Sciences, We would want to express our appreciation to the College of Agricultural Engineering Sciences for supplying the pomegranate transplant that was used in this study.

\section{References}

A. O. A. C. 1995. Officinal Methods of Analyses chemistry. 16th Ed. A.O.A.C. International, Gaithersburg, M. O.

A. O. A. C. 1975. Official Methods of Analyses Association of Official Analytical Chemists. Washington D. C., 12th Ed.
Al-Rawi, A. A. and A. Khalaf-Alla. 2000. Analysis of Experimental Agric. Design. Dar Al-Kutub for Printing and Publishing. Mosul Univ. Iraq. (In Arabic).

Arash O, H. Ansari nik and M. Ghazaghi. 2012. Prosopis Farcta beans increase HDL cholesterol and decrease LDL cholesterol in ostriches (Struthio camelus). Trop Anim Health Prod DOI 10.1007/s1 1250-012-0234-x

Direkvand-Moghadam F, Ghasemi-Seyed V, Abdali-Mashhadi A, Lotfi A and D. A. Moghadam and A. Delpisheh. 2014. Extraction and measurement of the quercetin flavonoid of Prosopis Farcta in Khouzestan climatic condition. Adv. Herb Med. 1(1):29-35.

Guil-Guerrero, J.L. R. Fuentes, M.M, M.E.T. Isasa. 2003. Fatty acids and carotenoids from Stinging Nettle (Urtica Dioica L.). J. Food Compos. Anal. 16, 111-119.

Houda B. L, H. Mejri, A. Feriani, Sh. Khemiri, E. Saadaoui, N. Nasri and N. Tlili. 2015. Prosopis Farcta Seeds: Potential Source of Protein and Unsaturated Fatty Acids?. J Am Oil Chem Soc DOI 10.1007/s11746-015-2660-1 IJPSR. 9(4): 13871396.

Lajnef HB, Mejri H, Feriani A, Khemiri S, Saadaoui E, Nasri N, N. Tlili. 2015. Prosopis Farcta Seeds: Potential Source of Protein and Unsaturated Fatty Acids?. J Am Oil Chem Soc. 92:1043-1050.

Mirdehghan, S.H. and M. Rahemi. 2007. Seasonal changes of mineral nutrient and phenolic in pomegranate (Punica granatum L.) fruit. Sci. Hortic. 111, 120-127.

Nar. Retrieved at 01.06. 2011 from www.batem.gov.tr/urunler/meyvelerimiz/nar/nar.h $\operatorname{tm}$

Olsen, S. R. and L. E. Semmers. 1982. Chemical and microbiological properties, 2nd ed, Amer. Soc. Agro. Madison, WI, USA. Phpsphorus: 403-430 In: A. L. Page, Methods of soil analysis, Agro. 9 (2).

Orcic, D.; Franciškovic, M.; Bekvalac, K.; Svircev, E.; Beara, I.; Lesjak, M.; Mimica-Dukic, N. 2014. Quantitative determination of plant phenolics in Urtica Dioica extracts by high-performance liquid chromatography coupled with tandem mass spectrometric detection. Food Chem. 143, 48-53. 
Otles, S., B. Yalcin. 2012. Phenolic compounds analysis of root, stalk, and leaves of nettle. Sci. World J.

Page, A. L.; R. H. Miller and O. R. Keeney. 1982. Methods of Soil Analysis. Part. Amer. Soc. Agro. Inc. Publisher, Madison, Wisconsin, U.S.A.

Pasiecznik, N. M.; Harris, P. J. C.; Smith, S. J. 2004. Identifying tropical Prosopis species-A field guide. HDRA, Coventry, UK.

Poudineh Z, Amiri R, Najafi S, N. Mir. 2015. Total phenolic content, antioxidant, and antibacterial activities of seed and pod of Prosopis Farcta from Sistan region, Iran Azar J. Agric. 2(2):51-56.

Repajic, M.; Cegledi, E.; Kruk, V.; Pedisic, S.; Çınar, F.; Bursac Kovǎcevic, D.; Zutic, I.; V. Dragovic-Uzelac. 2020. Accelerated Solvent Extraction as a Green Tool for the Recovery of Polyphenols and Pigments from Wild Nettle Leaves. Processes, 8, 803.

Rutto, L. K. , Xu Y., Ramirez E., and M. Brandt. 2013. Mineral properties and dietary values raw and processed Stinging nettle (Urtica dioica L.). Int. J. Food Sc. doi: $10.1155 / 2013 / 857120$.

Sajfrtova, M.; Sovova, H.; Opletal, L.; Bartlova, M. Near. 2005. critical extraction of sitosterol and scopoletin from stinging nettle roots. J. Supercrit. Fluids, 35, 111-118.

SAS, Institute. 2015. Base SAS 9.4 procedures guide. SAS Institute. USA.

Sheikh, M. K. and N. Manjula. 2009. Effect of split application of $\mathrm{N}$ and $\mathrm{K}$ on growth and fruiting in 